Original Research

\title{
Decreased Emotional Dysregulation Following Multi-modal Motion-assisted Memory Desensitization and Reprocessing Therapy (3MDR): Identifying Driving Factors in Remediation of Treatment-Resistant PTSD
}

Emily Tang ${ }^{1,2,7}$, Chelsea Jones ${ }^{2,3,4}$, Lorraine Smith-MacDonald ${ }^{1,2}$ Matthew R.G. Brown ${ }^{1,2}$, Eric H.G.J. Vermetten $^{3,5,6}$, Suzette Brémault-Phillips ${ }^{1,2,7 *}$

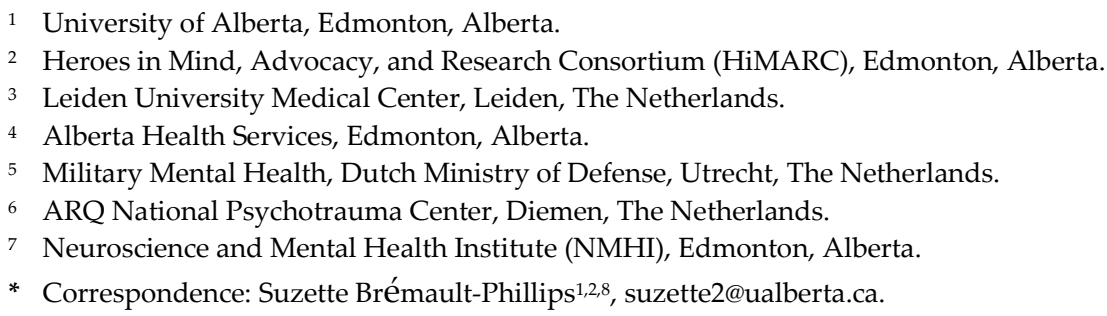

Abstract: Multi-modal Motion-assisted Memory Desensitization and Reprocessing Therapy (3MDR), an interactive, virtual-reality assisted, exposure-based intervention for PTSD, has shown promising results for treatment-resistant Posttraumatic Stress Disorder (TR-PTSD) among military members (MMs) and Veterans in Randomized Controlled Trials. Previous research has suggested that emotional regulation (ER) and emotional dysregulation (ED) may be factors which are correlated with symptom severity and maintenance of TR-PTSD. This embedded mixed-methods pilot study $(n=9)$ sought to explore the impact of 3MDR on ER and ED of MMs and Veterans. Difficulties in Emotional Regulation Scale (DERS-18) data was collected at baseline, prior to each session, and at 1 week, 1 month and 3 months post-intervention and analyzed using a Wilcoxon signed-ranks test. Qualitative data collected from sessions, debriefs, and follow-up interviews were transcribed and descriptively analyzed. Results demonstrated statistically significant decreases in DERS-18 scores from pre-intervention to postintervention at each timepoint. Qualitatively, participants perceived improvements in ER within specified DERS-18 domains. We describe how 3MDR's unique and novel approach may address ED through cognitive-motor stimulation, narration, divergent thinking, reappraisal of aversive stimuli, dual-task processing, and reconsolidation of traumatic memories. Further investigation is underway to better understand the underlying neurobiological mechanisms by which 3MDR addresses ER and PTSD.

Keywords: 3MDR; treatment-resistant PTSD; military; veterans; mental health; emotional regulation 


\section{Introduction}

Posttraumatic Stress Disorder (PTSD), one of the leading mental health disorders reported by military organizations worldwide, has detrimental effects such as attrition and overburdening of healthcare resources (Currier et al., 2014; Zamorski et al., 2016). Military members (MMs) are vulnerable to developing PTSD due to training and deployment experiences, and the stressful and often traumatic nature of their roles (Boulos and Zamorski, 2016; Vermetten and Ambaum, 2019). Symptoms of PTSD among MMs often include hypervigilance, reduced cognitive abilities, emotional dysregulation (ED), and comorbidities of anxiety and mood disorders (Bryant, 2019). MMs may also experience Moral Injury (MI) concurrently with PTSD, which is associated with feelings of shame, guilt, and distress following exposure to potentially morally injurious events (PMIEs) and violations of core morals and beliefs (Vermetten and Jetly, 2018; Nazarov et al., 2020).

Treatment approaches for PTSD among MMs and Veterans have variable effectiveness. Veterans see significantly worse treatment outcomes when compared with other populations that experience PTSD. Further, conventional treatment dropout rates for Veterans are as high as $78 \%$, with approximately $65 \%$ of patients continuing to meet the threshold for PTSD diagnosis following treatment (Nijdam and Vermetten, 2018; van Gelderen et al., 2018; Van Gelderen et al., 2020). Conventional evidence-based PTSD therapies, such as Cognitive Behavioral Therapy (CBT) and exposure therapies, are also less effective in the treatment of combat-related treatment resistant PTSD (TR-PTSD) subtype (Vermetten et al., 2013; van Gelderen et al., 2020). Therefore, there is a crucial need for novel and improved treatment approaches that address TR-PTSD among MMs and Veterans.

\subsection{MDR Intervention}

Multi-modal Motion-assisted Memory Desensitization and Reprocessing Therapy (3MDR) is a novel interactive psychotherapeutic approach for TR-PTSD (Nijdam and Vermetten, 2018; van Gelderen et al., 2018; Van Gelderen et al., 2020) that is delivered in a virtual reality environment. The intervention was initially developed in the Netherlands to treat MMs and Veterans experiencing TR-PTSD who had limited success with other forms of treatment. Combining aspects of conventional PTSD therapies, 3MDR includes Eye Movement Desensitization and Reprocessing Therapy (EMDR), Virtual Reality Exposure Therapy (VRET), and Trauma-focused CBT (Seidler and Wagner, 2006; Nijdam and Vermetten, 2018; van Gelderen et al., 2018; Van Gelderen et al., 2020), and introduces components such as a motor engagement via treadmill walking. Data from recently published RCTs (Bisson et. al, 2020; Van Gelderen et. al, 2020) demonstrate the efficacy of 3MDR therapy among MMs and Veteran populations who experience TRPTSD (Jones et al., 2020).

The 3MDR intervention consists of 10 sessions inclusive of selecting images and music, trauma processing and reconsolidation. 3MDR includes a pre-platform session (session 1), during which the patient selects and orders images and music. Symbolic representations in the form of images (i.e., photographs, sketches) related to his or her traumatic experiences are selected and ordered from least to most distressing. Music is also identified that both reminds the patient of the past time of trauma and facilitates the emotional memory network (i.e., a piece of music that is activating of the trauma), and supports a return to the present (i.e., a second contemporary piece that is soothing, compassionate, joyful). Platform sessions 2-7 are 90-minute therapy sessions in the virtual reality environment (VRE), including a 30-minute debrief. Each platform session involves 3 phases (pre-platform, platform, and post-platform). In the pre-platform phase, the therapist and patient confirm the order of images and music for the session. During the platform phase, the patient dons a safety harness and is accompanied by a 3MDR therapist while walking continuously on a treadmill at a self-selected pace. The patient first warms up by walking on the treadmill while listening to self-selected music 
connecting them to traumatic experiences and then, during each of seven 3-5 minute cycles, walks down a virtual "hallway" toward a self-selected trauma-related image. The patient describes the image, physical sensations, and feelings to the therapist. The therapist assists the patient with generating descriptive words/phrases which are then projected in front of the image on the screen. and which the patient is instructed to aloud. For a duration of 30 seconds thereafter, the patient then views a ball oscillating horizontally in the foreground of the image and words and reads out numbers as they appear on the ball. The patient cools down after the seventh cycle by walking while listening to self-selected music that facilitates re-connection to the present. Each session is concluded with a post-platform phase which includes debriefing, discussion, reconsolidation, and a mental wellness check/self-care plan. Sessions 8-10 are post-platform sessions focused on reconsolidation and contribute to meaning making of the acquired gains and emotional release. In-depth descriptions of 3MDR have been published elsewhere (van Gelderen et al., 2018; Jones et al., 2020).

Further investigation is required regarding the mechanisms by which 3MDR addresses PTSD and components of the intervention that differentiates its efficacy from other therapeutic interventions. One proposed mechanism relates to the manner in which 3MDR addresses emotional regulation (ER) and dysregulation (ED).

\subsection{Emotional Regulation and Dysregulation}

ER is the ability to adaptively respond to negative emotions that arise from threatening or aversive stimuli (Witte, 1992; Gross, 2015). As proposed by Witte in 1992, ER stems from the inability to directly cope with the presenting threat, resulting in a need to regulate emotions that stem from the experience. According to Gross (2015), ER consists of strategies that recognize and respond to negative emotions, such as cognitive reappraisal or redirection of attention from arousing stimuli, as well as inhibition and behavior modulation in response to emotion. These negative emotions include fear, anger, guilt, and shame (Lanius et al., 2010). ED conversely stems from an inability to perform tasks relating to ER. In PTSD, ED can present itself in the form of both emotional underand over-modulation (Yehuda et al., 2015). Emotional under-modulation involves hypervigilance, heightened experiences of negative emotions, and poor inhibition and behavioral adaptations in response to negative emotions. This subtype sees decreased frontal lobe activity, particularly in the medial prefrontal cortex (mPFC) and anterior cingulate cortex. On the other hand, emotional overmodulation takes the form of repression, extreme avoidance, inability to perceive one's emotions, and depersonalization and or derealization. The overmodulation subtype has excess $\mathrm{mPFC}$ and anterior cingulate activity. This study will focus on addressing the emotional undermodulation subtype, and ways in which it is potentially stabilized by 3MDR.

Associated with reduced ER (Bryant, 2019; Raudales et al., 2020; Spies et al., 2020), symptoms of PTSD include negative mood, cognitive deficits, avoidance, and hyperarousal (PTSD diagnostic Criterions D, C and E respectively). Recent studies suggest that difficulties with ER may exacerbate severity and maintain PTSD symptoms (Raudales et al., 2020; Spies et al., 2020). In particular, aspects of ED such as maladaptive or absent regulatory strategies, avoidance and repression of emotions, and or unawareness of emotions have been linked to PTSD symptom severity (Christ et al., 2021). Among MMs and Veterans, ED can have significant impacts on their ability to function in general and can potentially result in a decreased ability to effectively engage in and maintain employment and interpersonal relationships. It can also result in increased risk of harm to self, others, and the mission, and be disruptive to family, social and community functioning and quality of life. 


\subsection{Purpose}

This study aims to examine whether 3MDR is effective at improving ED, and identify potential neuropsychological mechanisms of 3MDR that may address ER, and consequently, TR-PTSD.

\section{Materials and Methods}

\subsection{Study Design}

This embedded mixed methods study (quantitative and qualitative) is a sub-study of a larger mixed-methods waitlist control randomized controlled trial exploring whether 3MDR is effective at addressing combat-related treatment-resistant PTSD among Canadian MMs and Veterans. This sub-study analyzed ER questionnaire data collected using the Difficulties in Emotional Regulation Scale (DERS-18), which is one of several self-reported questionnaires used to assess and monitor symptom changes across the 3MDR intervention. Transcribed data collected during participant sessions, debriefing, and/or post-intervention interviews was also used to analyze the functional impact of 3MDR on ED. The study has approval from the University Research Ethics Board and the Canadian Armed Forces (CAF) Surgeon General Research Program.

Two other papers from our group present analyses of certain aspects of the DERS18 data used in the current study (Jones et al. 2021a, 2021b). The analyses presented here are distinct from those in the other two papers.

\subsection{Sample Eligibility and Size}

A targeted study sample size of $40 \mathrm{MMs}$ and/or Veterans was set for the larger mixed-methods waitlist control randomized controlled trial to account for a 20 percent dropout rate and allow for power at 32 participants. With 4 latent variables, for $80 \%$ significance at a $5 \%$ significance level, the sample size required for this study is $24\left(\mathrm{R}^{2}=\right.$ 0.50) (Cohen, 1992). The sample used in the sub-study analyzing ER included data collected from 9 participants prior to a pause in data collection due to COVID-19 physical distancing restrictions.

\subsection{Inclusion and Exclusion Criteria}

3MDR study participants include regular and reserve CAF-MMs and Veterans aged 18-60 years under the care of a service provider associated with a Canadian Forces Base, Operational Stress Injury Clinic, or Veterans Affairs Canada. Participants met the Diagnostic and Statistical Manual 5th Edition (DSM5; American Psychiatric Association, 2013) criteria for PTSD diagnosis, had PTSD that was combat-related, had a score of 30 or higher on the Clinician-Administered PTSD Scale for DSM-5 Worst Month version, had been unsuccessful with at least 2 conventional PTSD treatments, and were stable on their current psychotropic medication for at least 4 weeks before entering the study. Individuals with co-morbidity were included if they satisfied the other inclusion/exclusion criteria. Participants were English-speaking and able to provide informed written consent. A detailed 3MDR protocol has previously been published (Jones et al., 2020).

\subsection{Recruitment and Setting}

Recruitment was conducted by word of mouth as convenience and snowball sampling. Service providers supporting MMs and Veterans, after being informed of the study via word of mouth and institutional email, informed patients who met the study inclusion and exclusion criteria. Potential participants were provided with a "Permission to Share Contact Information with the Research Team" form by their service provider. Completed forms were forwarded to a researcher who contacted potential participants via phone or email, provided further information about the study and determined 
eligibility to participate. Voluntary verbal and written informed consent were obtained from all CAF-MMs and veterans participating in the study.

The study uses the Computer Assisted Rehabilitation ENvironment (CAREN) system, located in the Glenrose Rehabilitation Hospital in Edmonton, Alberta. Quantitative data collection including self-report questionnaires, and qualitative data including transcripts of participant interviews, were collected pre-intervention, at each session, and at 1 week, 1 month, 3 months, and 6 months post-intervention.

\subsection{Questionnaire}

The DERS-18, a shortened yet comparative version of the DERS-36 found to effectively measure ER (Victor and Klonsky, 2016; Christ et al., 2021), was used in the study. Composed of 18 items, the DERS-18 is divided into six subsections: Awareness (lack of emotional awareness), Clarity (lack of emotional clarity), Goals (hindered goal-related activity), Impulse (lack of inhibition), Nonacceptance (nonacceptance of negative affective states), and Strategies (lack of effective coping strategies). Each subsection is assessed via three focused questions adopted from the DERS-36. Questions are rated on a 5-point Likert scale (Christ et al., 2021), with higher rating indicating greater experienced difficulty in ER. The three questions under the Awareness subsection are reverse scored. A high DERS-18 score indicates greater difficulty with ER, and a decrease in DERS-18 score suggests improvement of ED.

\subsection{Data Collection}

Quantitative: Participants were asked to complete a DERS-18 questionnaire prior to starting the 3MDR intervention (T0 baseline) (Jones et al., 2020). Following the intervention, DERS-18 data was collected at four follow-up timepoints: 1 week (T1), 1 month (T2), 3 months (T3), and 6 months (T4) post-intervention. Total DERS-18 scores from preintervention T0 were compared to post-intervention scores from T2, T3, and T4 to examine change in score following completion of 3MDR. Due to COVID-19 and other limitations, limited T4 scores were collected, and were therefore not included in the analysis. Missing data at varying time points resulted in only 5 participants having scores at all four pre- and post-intervention timepoints. To maximize statistical sensitivity of the analysis, any participant with both pre- and post-intervention scores for each of the three pairwise comparisons (T0 vs. T1, T0 vs. T2, T0 vs. T3) were utilized. Nine participants were used to compare T0 and T1 scores (P4, P5, P6, P8, P10, P11, P13, P16, P19), six participants to compare $\mathrm{T} 0$ and $\mathrm{T} 2$ scores (P4, P5, P8, P10, P13, P19), and six participants to compare T0 and T3 scores (P4, P5, P6, P8, P13, P19). A mean trendline was calculated using data from five participants ( $\mathrm{P} 4, \mathrm{P} 5, \mathrm{P} 8, \mathrm{P} 13, \mathrm{P} 19)$ from whom data was recorded at all three post-intervention timepoints (T3, T4, T5).

Qualitative: Audio and video recordings of data from 3MDR platform sessions, session debriefings, and $\mathrm{T} 1$ to $\mathrm{T} 4$ follow-up interviews were transcribed and deductively reviewed based on the domains of the DERS-18 for relevant changes in ER. Interview questions (e.g., How has 3MDR impacted your quality of life, relationships, social integration/engagement, and other meaningful activities or life skills?") probed changes in ER following 3MDR.

\subsection{Data Analysis}

Changes in DERS-18 scores were analyzed in IBM SPSS using a Wilcoxon signedranks test to compare participant pre-intervention baseline scores and post-intervention scores. Participants with pre-intervention baseline scores (T0) were compared with their respective post-intervention scores (T1, T2, T3). The Wilcoxon test is a non-parametric test designed to compare related samples (Taheri and Hesamian, 2013). For each of the three pairwise comparisons, a mean, mean rank, Z score, and p-value were collected to analyze for changes in DERS-18 scores.

Qualitative data was deductively analyzed using descriptive analysis. The rationale behind selecting a deductive descriptive analysis approach was to compare and 
triangulate the quantitative results found with the DERS-18. Specifically, we sought to explore if participants noticed and/or experienced a tangible change within their ER which they would attribute to 3MDR. Qualitative descriptive analysis is a widely cited research tradition and has been identified as important and appropriate for research questions focused on discovering the who, what, and where of events or experiences and gaining insights from informants regarding a less understood phenomenon (Polit and Beck, 2009). It is also the label of choice when a straight description of a phenomenon is desired or information is sought to develop and refine questionnaires or interventions (Neergaard et al., 2009).

A concurrent parallel approach following a data transformation model was utilized in the data analysis process to converge the data for comparing and contrasting the quantitative statistical results with qualitative findings (Creswell et al., 2011; Schoonenboom \& Johnson, 2017).

\section{Results}

\subsection{DERS-18 Score 1 Week Post-Intervention (T0 vs. T1)}

Mean participant total DERS-18 score 1 week following completion of 3MDR intervention (T1) was shown to decrease from baseline pre-intervention mean participant score (Table 2). The Wilcoxon test generated a statistically significant p-value of 0.021 $(\mathrm{N}=9, \mathrm{Z}=-2.314)$.

\subsection{DERS-18 Score 1 Month Post-Intervention (T0 vs. T2)}

Mean participant DERS-18 score 1 month following completion of 3MDR intervention was also shown to decrease from the baseline pre-intervention mean participant score (Table 2). The conducted Wilcoxon test generated a statistically significant $\mathrm{p}$-value of $0.028(\mathrm{~N}=6, \mathrm{Z}=-2.201)$.

\subsection{DERS-18 Score 3 Month Post-Intervention (T0 vs. T3)}

Mean participant DERS-18 score 3 months post-intervention also showed a decrease from the baseline pre-intervention mean participant score (Table 2). The Wilcoxon test generated a statistically significant $\mathrm{p}$-value of $0.027(\mathrm{~N}=6, \mathrm{Z}=-2.207)$.

Decreases in DERS-18 score across time are summarized in Table 2 and Figure 1.

Table 1. Wilcoxon test results. All three comparisons reject the null hypothesis, indicating statistically significant decrease post-intervention from baseline pre-intervention DERS-18 score.

\begin{tabular}{|l|l|l|l|l|l|l|}
\hline & \multicolumn{2}{l|}{$\mathbf{1}$ WEEK } & $\mathbf{1}$ MONTH & \multicolumn{2}{l|}{ 3 MONTHS } \\
\hline & T0 & T1 & T0 & T2 & T0 & T3 \\
\hline Mean & 56.67 & 49.56 & 52.00 & 41.33 & 54.33 & 40.67 \\
\hline df & 8 & 5 & 5 & 0.027 \\
\hline p-value & 0.021 & 0.028 & & -2.207 \\
\hline Z score & -2.314 & -2.201 & & \\
\hline
\end{tabular}

Figure 1. Changes in DERS-18 scores in participants across time. T0 denotes pre-intervention baseline. T1, T2, and T3 denote post-intervention follow-up at 1 week, 1 month, and 3 months. Mean trendline (red dotted line) was calculated using five participants (P4, P5, P8, P13, P19) that had all three post-intervention timepoints recorded (T1, T2, T3). 


\section{Change in DERS-18 Score Over Post-Intervention Time Points}

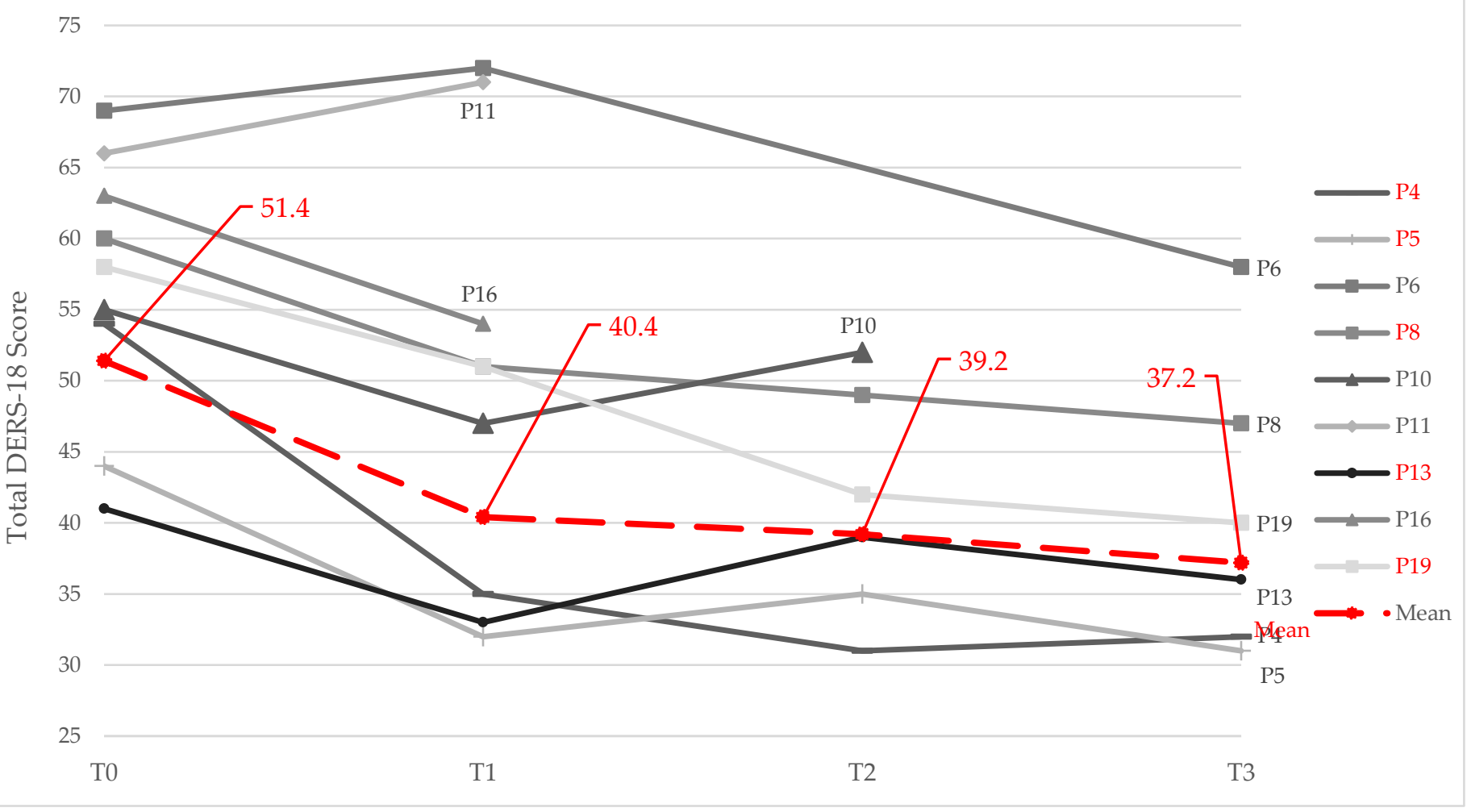

\subsection{Qualitative Changes in Participant Emotional Regulation}

Quotes collected from transcribed participant data are displayed in Table 3 and categorized based on relevance to DERS-18 subsections. The qualitative descriptive analysis illustrated that participants expressed a noticeable betterment in all DERS-18 subsections, most significantly in awareness, clarity, nonacceptance, and strategies. Participants expressed that gaining awareness of their emotions, feelings, sensations; having the correct and appropriate vocabulary to correctly identify these emotions, feelings, and sensations, and then finding strategies (including acceptance) was central to their treatment and the success of 3MDR. 
Table 2. Qualitative Narrative Analysis: Participant quotes describing changes in ER following 3MDR intervention.

\begin{tabular}{|c|c|}
\hline & Quotes \\
\hline $\begin{array}{l}\text { Range and } \\
\text { layers of emo- } \\
\text { tions ad- } \\
\text { dressed by } \\
3 \text { MDR }\end{array}$ & $\begin{array}{l}\text { "II]t's a complete } 180 \text { in perspective, I am no longer focused on trying to deal with anger and } \\
\text { whatever. I am focused on the guilt and shame that caused the anger to begin with. I have } \\
\text { gotten to an underlying layer of things... helped me realize it was real, to look at it in a } \\
\text { different manner. Shift from anger to shame and guilt which is actually what I had to face. } \\
\text { And I had never faced it until now." (P10) }\end{array}$ \\
\hline $\begin{array}{l}\text { Subsection of } \\
\text { DERS-18 }\end{array}$ & \\
\hline Awareness & $\begin{array}{l}\text { "I have noticed [positive] differences. Just being more aware of how I feel. And being able to, } \\
\text { you know, once I identify it I'm able to change the way I - what I'm thinking about. And } \\
\text { that's progress for me because that's always been a tough one... [to shift out of] an intru- } \\
\text { sive thought or negative state." (P5) } \\
\text { "My emotional range has improved. I don't avoid stuff anymore, I kind of focus on it, try to } \\
\text { deal with it." (P2) } \\
\text { "Being more patient. Knowing my reactions. My triggers. What triggers me off, and what } \\
\text { sets me off easier. Noticing little things, I used to get so emotionally upset." (P3) }\end{array}$ \\
\hline Clarity & $\begin{array}{l}\text { "I don't think I've expanded my vocabulary. I think I've just learned to put words to feel- } \\
\text { ings...I'm more connected to my emotions and my feelings and my thoughts." (P5) }\end{array}$ \\
\hline Goals & $\begin{array}{l}\text { "My focus has improved, concentration has improved. Go to work and do paperwork. I still } \\
\text { tire quickly when I have to pay attention to details; it does wear me out, but at least I can } \\
\text { do it now. Where before I wouldn't have been able to... improvement is there but it's not } \\
100 \% . "(P 2)\end{array}$ \\
\hline Impulse & $\begin{array}{l}\text { "I'm a lot calmer, I'm allowing things to happen without reacting, with the kids and stuff. } \\
\text { Stomp, stomp, pound, smash. I'm not letting it get to me, 90\% better. I still get irked. You } \\
\text { get that twitch. Even my driving is getting better. [Less] reacting to the morons in front, } \\
\text { behind and on the side of me." (P13) }\end{array}$ \\
\hline $\begin{array}{l}\text { Nonac- } \\
\text { ceptance }\end{array}$ & $\begin{array}{l}\text { "In week three, and again in post-week five, was a little bit less of beating myself up, a little bit } \\
\text { of saying 'it's okay to take care of yourself, it's okay to put yourself in front sometimes'." } \\
\text { (P11) } \\
\text { "I used to be very ashamed of [my PTSD]. Very.... [Now] everybody I know knows that I suf- } \\
\text { fer from it." (P6) }\end{array}$ \\
\hline Strategies & $\begin{array}{l}\text { "Usually... I try to deal with [negative thoughts and emotions] in a healthy manner. Spend } \\
\text { time with my cat or do photography or just do something different" (P5) } \\
\text { "[Prior to } 3 M D R \text {, arguments with wife] would carry on for at least an hour or two. And it } \\
\text { would take me 2-3 hours to calm down from that. And you know I still get some of those } \\
\text { rushes I guess. But not as bad as they used to be." (P6) }\end{array}$ \\
\hline
\end{tabular}

\section{Discussion}

This study examined whether 3MDR is effective at improving ED among MMs and Veterans with TR-PTSD. The results demonstrated a statistically significant decrease in DERS-18 scores (Table 2, Figure 1) for all three pre/post-intervention comparisons, with 
the mean difference in the DERS-18 scores increasing over time. This suggests that ED is improved following 3MDR intervention and signifies potential efficacy of 3MDR in addressing difficulties with ER, as originally hypothesized. 3MDR's multi-modal and graded, stage-based approach may uniquely account for these positive effects. Qualitative data from the deductive descriptive analysis also indicate noticeable improvement in ER within the subsections of the DERS-18, supporting the quantitative results, with participants reporting noticeable changes in functioning across numerous domains of ER in their day-to-day lives. These positive preliminary results regarding 3MDR's impact on ER in trauma affected populations is encouraging.

Several potential mechanisms of 3MDR may facilitate changes in ER. These include cognitive-motor stimulation, eye movement bilateral stimulation, and comprehensive treatment addressing ER.

\subsection{Cognitive-Motor Stimulation}

Cognitive-affective and motor systems are functionally interconnected, and subconsciously influence one another (Oppezzo and Schwartz, 2014; Pietrzak et al., 2018; van Gelderen et al., 2018). Body language and motor actions are thought to impact cognitive perception and emotional valence. Neural circuits link sensorimotor regions with diffuse projections (Guell et al., 2017), such as the cerebellum, striatum, and thalamus, with cognitive-affective areas such as the frontal lobe. The walking motor engagement may act in a bottom-up fashion to guide cognitive and affective appraisal of presented stimuli during 3MDR, potentially controlling ED. These systems are goal-oriented and aim to align stimulus perception and resultant behavior. When there is inconsistency between appraisal and action, the cognitive-affective system, based on the idea of cognitive dissonance, is more inclined to adjust in order to resolve this conflict (Kitayama et al., 2013). Resolution of cognitive dissonance recruits self-mediating and emotionallyregulating regions such as the medial prefrontal cortex (mPFC), posterior cingulate cortex, and precuneus (Kitayama et al., 2013). Directionality of movement can also impact appraisal of stimuli to shift more positively or negatively (Pietrzak et al., 2018). Therefore, it is hypothesized that the walking approach during 3MDR towards a traumatogenic stimulus may induce a positive shift in appraisal and divergent thinking, reducing avoidance and intensity of negative emotions and perceptions. Cognitive-motor stimulation has previously been studied in relation to ER. Pietrzak et al. (2018) addressed ED through controlling breathing patterns, speech tone and content, and posture during CBT. Consequently, stimulating neural networks linking cognitive-affective and sensorimotor function during 3MDR may elevate ER through this proprioceptive feedback.

\subsection{Eye Movement Bilateral Stimulation}

The EMDR working memory task during 3MDR aims to engage neural networks that may both enable the reduction of or disengagement from negative emotions and the fear response and set the stage for reconsolidation of traumatic memories. The working memory task, which has elements of EMDR given its use of eye movements in a sequential manner, is enhanced in 3MDR with the speaking aloud of numbers appearing on an oscillating ball. EMDR is hypothesized to promote neuroplasticity in regulatory regions related to emotional processing (Landin-Romero et al., 2018), such as increased right side intra-hemispheric connectivity. Similarly, EMDR facilitates heightened activity in motor regions involved in the cognitive-motor feedback loop. Areas of the frontal lobe, including the medial and dorsolateral prefrontal cortex (Herkt et al., 2014; Giotakos, 2020), are stimulated during EMDR, which have been found to be responsible for fear inhibition, fear extinction learning, and ER. These regions have also been implicated to be downregulated in PTSD (Giotakos, 2020). The integration of the EMDR task in 3MDR intervention may allow sequential engagement and disengagement from high emotional memories and contribute to a reduction of the valence of negative emotions that result in a need for ER, thereby indirectly addressing ED. 


\subsection{Comprehensive Treatment Addressing Emotional Regulation}

Underlying the efficacy of the 3MDR intervention in regulating ED may be the combination of treatment methods (treadmill walking, EMDR, and exposure therapy). Difficulties with ER are highly correlated with severity and maintenance of TR-PTSD (Raudales et al., 2020; Spies et al., 2020). 3MDR may facilitate ER in that, layer by layer, dysregulated emotions ranging from intense sadness, grief, loss, or anger, shame, betrayal or guilt, are addressed in the course of treatment. Affective reconsolidation occurs by correcting and tackling avoidance. Further, experiencing, releasing, naming and processing raw dysregulated emotions in a safe, predictable context and therapeutic relationship facilitates new meaning-making, memory reconsolidation, and the overlay of newfound memories and experiences over traumatic ones.

3MDR's comprehensive and multi-modular approach may affect several neurobiological systems. An immersive, virtual-reality supported exposure-based therapy, 3MDR may recruit and stimulate prefrontal regions, specifically the $\mathrm{mPFC}$, that are dampened in the emotional under-modulation subtype. Through engaging these areas, 3MDR may facilitate proper inhibition of the amygdala and balance of the amygdalar-prefrontal feedback loop. Further, one of the most significant neural correlates of PTSD is hyperactivation of the amygdala (Sripada et al., 2012; Giotakos, 2020), which maintains a feedback loop with the regulatory $\mathrm{mPFC}$. The $\mathrm{mPFC}$ is related to self-regulation, such as processing and memory of emotions, and sends inhibitory projections to the amygdala, which is responsible for detection of threats, fear conditioning, and emotional intensity (Sripada et al., 2012). The exposure therapy component in 3MDR involves approaching and engaging with traumatic memories that elicit fear, to effectuate fear extinction learning. Fear extinction requires the $\mathrm{mPFC}$ for storage of fear extinction memory to appropriately modulate amygdalar activation (Morgan et al., 1993; Milad and Quirk, 2002). Hyperactivity of the amygdala reciprocally inhibits mPFC activation (Shin et al., 2004; Andrewes and Jenkins, 2019; Ebneabbasi et al., 2020), which can further attenuate mPFC regulation. In PTSD, hyperactivity of the amygdala and decreased white matter prefrontal-amygdalar connectivity is positively correlated with symptom severity (Shin et al., 2004; Andrewes and Jenkins, 2019). Absence of of top-down control by the mPFC hinders fear extinction learning in PTSD (Andrewes and Jenkins, 2019), resulting in heightened arousal, and reduced ER (Sripada et al., 2012; Andrewes and Jenkins, 2019). Efficacy of 3MDR in treating difficulties with ER may be derived through extrinsically induced upregulation of the $\mathrm{MPFC}$ to facilitate emotional regulatory processes and reduce intensity and negative appraisal of the traumatic memories (fear extinction).

ED associated with both PTSD and MI may also be addressed through 3MDR. Exposure to morally injurious experiences, which frequently overlaps with exposure to traumatic experiences associated with combat related TR-PTSD, is a high-risk factor for the development of MI (Vermetten and Ambaum, 2019; Nazarov et al., 2020), and the comorbidity of MI and PTSD can lead to TR-PTSD. Protopopescu et al. (2020) suggest that MI severity is positively correlated with severity of cluster C and D PTSD symptoms (avoidance and negative alterations in cognition and mood; APA, 2013). Although MI is associated with PTSD, it differs in neuropathology (Spies et al., 2020), and therefore may require alternative treatment approaches. Therefore, addressing ER may be effective in treating MI in addition to PTSD, and may explain 3MDR's efficacy over other conventional therapeutic approaches. Treatment of MI through 3MDR may further remediate TR-PTSD symptoms, due to the interconnected nature of the two.

\subsection{Future Research}

3MDR, a promising innovative therapeutic intervention that has generated statistically significant outcomes, warrants much further study and real-world application with trauma affected populations. The effect of active participation and engagement of patients by way of walking while simultaneously talking and performing tasks is an area of further investigation. Exploring underlying therapeutic principles and mechanisms 
involved in 3MDR that individually and collectively address various layers of ED is also an area requiring further study. To further investigate hypothesized neurobiological mechanisms driving efficacy of 3MDR, and validate the findings from a biological perspective, the study would benefit from implementation of EEG or other neuroimaging techniques to analyze neuroactivity during, pre- and post-intervention. Continuation of the study and recruitment of further participants will allow more data collection and analysis, enabling greater sample sizes and improving validity of the results. In particular, generating more 6-month post-intervention (T4) data would allow for deeper insight on long-term effects of 3MDR on ER.

\subsection{Limitations}

This study was positioned to assess data in a larger cohort. We experienced limitations, however, on data collection and analysis due to COVID-19 restrictions. As analysis of this data sets the stage for new studies, we did not want to hold back on analysis of this dataset. We do need to report that, despite these data indicating statistically significant and promising changes in ER following 3MDR, the small sample size limits the validity and generalizability of the results. Ongoing data collection and analysis would allow for determination of stronger conclusions regarding the efficacy of 3MDR on addressing ED.

\section{Conclusions}

This study reports enhanced ER on a sample of military members and Veterans $(n=9)$ with combat-related TR-PTSD following 3MDR, with positive statistically significant preliminary outcomes lasting months following the intervention. It is hypothesized that 3MDR's comprehensive, multi-modal treatment approach is responsible for the active engagement of suppressed stress regulatory circuits and stimulation of neurological regions (e.g., $\mathrm{mPFC}$, amygdala) pertinent in emotional processing and fear inhibition. These may be underlying mechanisms by which 3MDR addresses cognitive avoidance and ED. Continuing research on this novel intervention's ability to enhance ER in trauma affected populations is warranted. The potential impact of 3MDR on ER is not only a promising development for TR-PTSD, but also for other mental health challenges, such as MI, that may contribute to ED. It remains to be seen if individuals from diverse other uniformed and civilian populations struggling with trauma-based mental health conditions may benefit equally from the 3MDR intervention.

Supplementary Materials: The following are available online at www.mdpi.com/xxx/s1, Figure S1: Change in DERS-18 scores in participants across time, Table S1: Wilcoxon test results; Table S2: Qualitative Narrative Analysis.

Author Contributions: Conceptualization, C.J., L.S-M., M.B., E.V., E.T. and S.B-P.; methodology, C.J. and S.B-P.; formal analysis, E.T., C.J., L.S-M., M.B., E.V. and S.B-P.; investigation, C.J., L.S-M., S.B-P.; resources, S.B-P.; data curation, C.J., L.S-M., M.B. and S.B-P; writing-original draft preparation, E.T.; writing - review and editing, E.T., C.J., L.S-M., M.B., E.V., and S.B-P.; visualization, E.T., C.J., M.B.; supervision, C.J., L.S-M, S.B-P.; project administration, C.J., L.S-M., M.B., S.B-P.; funding acquisition, C.J., L.S-M, M.B and S.B-P.

Funding: This research was funded by the Government of Canada Innovation for Defence Excellence and Security (IDEaS) grant number CPCA-0617; Government of Alberta grant number 011427; the Glenrose Rehabilitation Hospital Foundation, grant number RES0042203; and the Royal Canadian Legion, grant numbers RES0048730 and RES0046384.

Institutional Review Board Statement: The study was conducted according to the guidelines of the Declaration of Helsinki and approved by the University of Alberta Research Ethics Board (Pro00084466, January 21, 2019) and the Canadian Armed Forces (CAF) Surgeon General Research Program (E2019-02-250-003-0003, April 1, 2019).

Informed Consent Statement: Informed consent was obtained from all subjects involved in the study. 
Data Availability Statement: The data presented in this study are available on request from the corresponding author. The data are not publicly available due to its sensitive nature and privacy limitations.

\begin{abstract}
Acknowledgments: The research team would like to acknowledge that this research has been supported by the Royal Canadian Legion AB/NWT Command; Government of Alberta Grant; Glenrose Rehabilitation Hospital Foundation; Government of Canada Innovation for Defence Excellence and Security (IDEaS) Grant and the University of Alberta. The research team would also like to thank study participants for sharing their time and experiences, the funders for enabling the study, and the Glenrose Rehabilitation Hospital, Canadian Armed Forces, Alberta Health Services, the Royal Canadian Legion AB/NWT, Veterans Affairs Canada, Dutch Ministry of Defense and Leiden University for their continued support.
\end{abstract}

Conflicts of Interest: 3MDR was first created by the Dutch Ministry of Defense and Professor Vermetten but does not stand to benefit financially were it to be adopted into routine clinical practice. The other authors have no potential conflicts of interest to declare.

\title{
References
}

American Psychiatric Association. Diagnostic and Statistical Manual of Mental Disorders. 5th ed. Arlington, VA. 2013.

Andrewes DG, Jenkins LM (2019) The Role of the Amygdala and the Ventromedial Prefrontal Cortex in Emotional Regulation: Implications for Post-traumatic Stress Disorder. Neuropsychol Rev.

Bisson J, van Deursen R, Hannigan B, Kitchiner N, Barawi K, Jones K, et al. (2020). Randomized controlled trial of multi-modular motion-assisted memory desensitization and reconsolidation (3MDR) for male military veterans with treatment-resistant post-traumatic stress disorder. Acta Psychiatr Scand. 142(2):141-151. DOI: 10.1111/acps.13200

Boulos D, Zamorski MA (2016) Military Occupational Outcomes in Canadian Armed Forces Personnel with and without Deployment-Related Mental Disorders. Can J Psychiatry 61:348-357 Available at: https://pubmed.ncbi.nlm.nih.gov/27254844.

Bryant RA (2019) Post-traumatic stress disorder: a state-of-the-art review of evidence and challenges. World Psychiatry 18:259-269.

Creswell JW, Plano Clark VL (2011) Designing and conducting mixed methods research, pp 58-88. Thousand Oaks, CA: SAGE Publications.

Christ NM, Elhai JD, Forbes CN, Gratz KL, Tull MT (2021) A machine learning approach to modeling PTSD and difficulties in emotion regulation. Psychiatry Res 297:113712 Available at: https://doi.org/10.1016/j.psychres.2021.113712.

Cohen, J. A power primer: Quantitative methods in psychology, 1992;112(1):155-159.

Currier JM, Holland JM, Drescher KD (2014) Residential treatment for combat-related posttraumatic stress disorder: identifying trajectories of change and predictors of treatment response. PLoS One 9:e101741-e101741 Available at: https://pubmed.ncbi.nlm.nih.gov/25058403.

Ebneabbasi A, Mahdipour M, Nejati V, Li M, Liebe T, Colic L, Leutritz AL, Vogel M, Zarei M, Walter M, Tahmasian M (2020) Emotion processing and regulation in major depressive disorder: A 7T resting-state fMRI study. Hum Brain Mapp n/a Available at: https://doi.org/10.1002/hbm.25263.

Giotakos O (2020) Neurobiology of emotional trauma. Psychiatriki 31:162-171.

Gross JJ (2015) The Extended Process Model of Emotion Regulation: Elaborations, Applications, and Future Directions. Psychol Inq 26:130-137 Available at: https://doi.org/10.1080/1047840X.2015.989751.

Guell X, Gabrieli J, Schmahmann J (2017) Embodied cognition and the cerebellum: Perspectives from the Dysmetria of Thought and the Universal Cerebellar Transform theories. Cortex 100.

Herkt D, Tumani V, Grön G, Kammer T, Hofmann A, Abler B (2014) Facilitating Access to Emotions: Neural Signature of EMDR Stimulation. PLoS One 9:e106350 Available at: https://doi.org/10.1371/journal.pone.0106350.

Jones C, Smith-MacDonald L, Miguel-Cruz A, Pike A, van Gelderen M, Lentz L, Shiu MY, Tang E, Sawalha J, Greenshaw A, Rhind SG, Fang X, Norbash A, Jetly R, Vermetten E, Brémault-Phillips S (2020) Virtual Reality-Based Treatment for Military Members and Veterans With Combat-Related Posttraumatic Stress Disorder: Protocol for a Multimodular Motion-Assisted Memory Desensitization and Reconsolidation Randomized Controlled Trial. JMIR Res Protoc 9:e20620 Available at: http://www.researchprotocols.org/2020/10/e20620/.

Jones, C., Lentz, L., Smith-MacDonald, L., Miguel-Cruz, A., Brémault-Phillips, S. (2021a). Tackling trauma with technology: Treating chronic combat-related PTSD in Canadian Armed Forces personnel and veterans with 3MDR, Annual Review of CyberTherapy and Telemedicine, 18; 235-239. https://www.arctt.info/volume-18-summer-2020

Jones, C., Smith-MacDonald, L, Brown, M R G, Pike, A, Vermetten, E, Brémault-Phillips, S (2021b, in review). Quantitative changes in mental health measures with 3MDR treatment for Canadian military members and veterans with treatment-resistant posttraumatic stress disorder, Brain and Behaviour.

Kitayama S, Chua HF, Tompson S, Han S (2013) Neural mechanisms of dissonance: an fMRI investigation of choice justification. Neuroimage 69:206-212 Available at: http://europepmc.org/abstract/MED/23238432.

Landin-Romero R, Moreno-Alcazar A, Pagani M, Amann BL (2018) How Does Eye Movement Desensitization and Reprocessing Therapy Work? A Systematic Review on Suggested Mechanisms of Action . Front Psychol 9:1395 Available at: https://www.frontiersin.org/article/10.3389/fpsyg.2018.01395. 
Lanius RA, Frewen PA, Vermetten E, Yehuda R (2010) Fear conditioning and early life vulnerabilities: Two distinct pathways of emotional dysregulation and brain dysfunction in PTSD. European Journal of Psychotraumatology 1:5467.

Milad MR, Quirk GJ (2002) Neurons in medial prefrontal cortex signal memory for fear extinction. Nature 420:70-74 Available at: http://europepmc.org/abstract/MED/12422216.

Morgan MA, Romanski LM, LeDoux JE (1993) Extinction of emotional learning: Contribution of medial prefrontal cortex. Neurosci Lett 163:109-113 Available at: http://www.sciencedirect.com/science/article/pii/030439409390241C.

Nazarov A, Fikretoglu D, Liu A, Richardson JD, Thompson M (2020) Help-seeking for mental health issues in deployed Canadian Armed Forces personnel at risk for moral injury. Eur J Psychotraumatol 11:1729032 Available at: https://pubmed.ncbi.nlm.nih.gov/32194921.

Neergaard MA, Olesen F, Andersen RS, Sondergaard J (2009) Qualitative description - the poor cousin of health research? BMC Medical Research Methodology 9.

Nijdam MJ, Vermetten E (2018) Moving forward in treatment of posttraumatic stress disorder: Innovations to exposure-based therapy. Eur J Psychotraumatol 9.

Oppezzo M, Schwartz DL (2014) Give your ideas some legs: The positive effect of walking on creative thinking. J Exp Psychol Learn Mem Cogn 40:1142-1152.

Pietrzak T, Lohr C, Jahn B, Hauke G (2018) Embodied cognition and the direct induction of affect as a compliment to cognitive behavioural therapy. Behav Sci (Basel) 8.

Polit DF, Beck CT (2012) Nursing research: Generating and assessing evidence for nursing practice. Wolters Kluwer, Lippincott Williams \& Wilkins.

Protopopescu A, Boyd JE, O'Connor C, Rhind S, Jetly R, Lanius RA, McKinnon MC (2020) Moral injury in Canadian military members and Veterans: Implications for military and healthcare sector response during the COVID-19 pandemic. J Mil Veteran Fam Heal COVID-19:Author's origin.

Raudales AM, Preston TJ, Albanese BJ, Schmidt NB (2020) Emotion dysregulation as a maintenance factor for posttraumatic stress symptoms: The role of anxiety sensitivity. J Clin Psychol:1-15.

Schoonenboom J, Johnson RB (2017) How to construct a mixed methods research design. KZfSS Kölner Zeitschrift für Soziologie und Sozialpsychologie 69:107-131.

Seidler GH, Wagner FE (2006) Comparing the efficacy of EMDR and trauma-focused cognitive-behavioral therapy in the treatment of PTSD: A meta-analytic study. Psychol Med 36:1515-1522.

Shin LM, Orr SP, Carson MA, Rauch SL, Macklin ML, Lasko NB, Peters PM, Metzger LJ, Dougherty DD, Cannistraro PA, Alpert NM, Fischman AJ, Pitman RK (2004) Regional cerebral blood flow in the amygdala and medial prefrontal cortex during traumatic imagery in male and female Vietnam veterans with PTSD. Arch Gen Psychiatry 61:168-176 Available at: http://europepmc.org/abstract/MED/14757593.

Spies JP, Cwik JC, Willmund GD, Knaevelsrud C, Schumacher S, Niemeyer H, Engel S, Küster A, Muschalla B, Köhler K, Weiss D, Rau H (2020) Associations Between Difficulties in Emotion Regulation and Post-Traumatic Stress Disorder in Deployed Service Members of the German Armed Forces. Front Psychiatry 11:1-15.

Sripada RK, King AP, Garfinkel SN, Wang X, Sripada CS, Welsh RC, Liberzon I (2012) Altered resting-state amygdala functional connectivity in men with posttraumatic stress disorder. J Psychiatry Neurosci 37:241-249 Available at: https://pubmed.ncbi.nlm.nih.gov/22313617.

Taheri SM, Hesamian G (2013) A generalization of the Wilcoxon signed-rank test and its applications. Stat Pap 54:457-470 Available at: https://doi.org/10.1007/s00362-012-0443-4.

van Gelderen MJ, Nijdam MJ, Haagen JFG, Vermetten E (2020) Interactive Motion-Assisted Exposure Therapy for Veterans with Treatment-Resistant Posttraumatic Stress Disorder: A Randomized Controlled Trial. Psychother Psychosom 89:215-227 Available at: https://www.karger.com/DOI/10.1159/000505977.

van Gelderen MJ, Nijdam MJ, Vermetten E (2018) An Innovative Framework for Delivering Psychotherapy to Patients With Treatment-Resistant Posttraumatic Stress Disorder: Rationale for Interactive Motion-Assisted Therapy. Front Psychiatry 9:176 Available at: https://www.frontiersin.org/article/10.3389/fpsyt.2018.00176.

Vermetten E, Ambaum J (2019) Exposure to combat and deployment; reviewing the military context in the Netherlands. Int Rev Psychiatry 31:49-59.

Vermetten E, Jetly R (2018) A Critical Outlook on Combat-Related PTSD: Review and Case Reports of Guilt and Shame as Drivers for Moral Injury. Mil Behav Heal 6:156-164 Available at: https://doi.org/10.1080/21635781.2018.1459973.

Vermetten E, Meijer L, van der Wurff P, Mert A (2013) The effect of military motion-assisted memory desensitization and reprocessing treatment on the symptoms of combat- related post traumatic stress disorder: First preliminary results. Annu Rev CyberTherapy Telemed 11:125-128 Available at: https://login.ezproxy.library.ualberta.ca/login?url=https://search.ebscohost.com/login.aspx?direct=true\&db=edscal\&AN=edscal.28393087\&site=eds-live\&scope=site.

Victor SE, Klonsky ED (2016) Validation of a Brief Version of the Difficulties in Emotion Regulation Scale (DERS-18) in Five Samples. J Psychopathol Behav Assess 38:582-589.

Witte K (1992) Putting the fear back into fear appeals: The extended parallel process model. Commun Monogr 59:329-349.

Yehuda R, Hoge CW, McFarlane AC, Vermetten E, Lanius RA, Nievergelt CM, Hobfoll SE, Koenen KC, Neylan TC, Hyman SE (2015) Post-traumatic stress disorder. Nat Rev Dis Prim 1:15057 Available at: https://doi.org/10.1038/nrdp.2015.57.

Zamorski MA, Bennett RE, Rusu C, Weeks M, Boulos D, Garber BG (2016) Prevalence of Past-Year Mental Disorders in the Canadian Armed Forces, 2002-2013. Can J Psychiatry 61:26S-35S Available at: https://pubmed.ncbi.nlm.nih.gov/27270739. 